\title{
Shared Ride Trip Planning with Geosensor Networks
}

\author{
Stephan Winter ${ }^{1}$, Silvia Nittel ${ }^{2}$ \\ ${ }^{1}$ Department of Geomatics, \\ The University of Melbourne, Australia \\ winter@unimelb.edu.au \\ ${ }^{2}$ Department of Spatial Information Science and Engineering, \\ University of Maine, USA \\ silvia@spatial.maine.edu
}

\begin{abstract}
We propose and investigate a model for shared ride trip planning in ad-hoc mobile geosensor networks. Our focus is on the communication strategies between the network nodes. In a dynamically changing network of autonomous nodes all trip plans and provisions need to be kept up-to-date. At the same time, energy consumption by broadcasting messages needs to be minimized. Hence, we have to solve an optimization problem: find an efficient communication strategy that still guarantees planning of acceptable trips in a continuously changing environment.
\end{abstract}

\section{INTRODUCTION}

Imagine Hillary who just has missed her bus to work today. Around Hillary the traffic is floating. Now she is glad to have subscribed to a service that mediates between her current travel needs and vehicles going into her direction in an ad-hoc manner. Hillary switches on her portable hand-held device, and it starts negotiating with devices in the vehicles close-by. It determines and books an optimal offer, and soon after Hillary sees a friendly car driver stopping for sharing a ride. The ride takes her a first

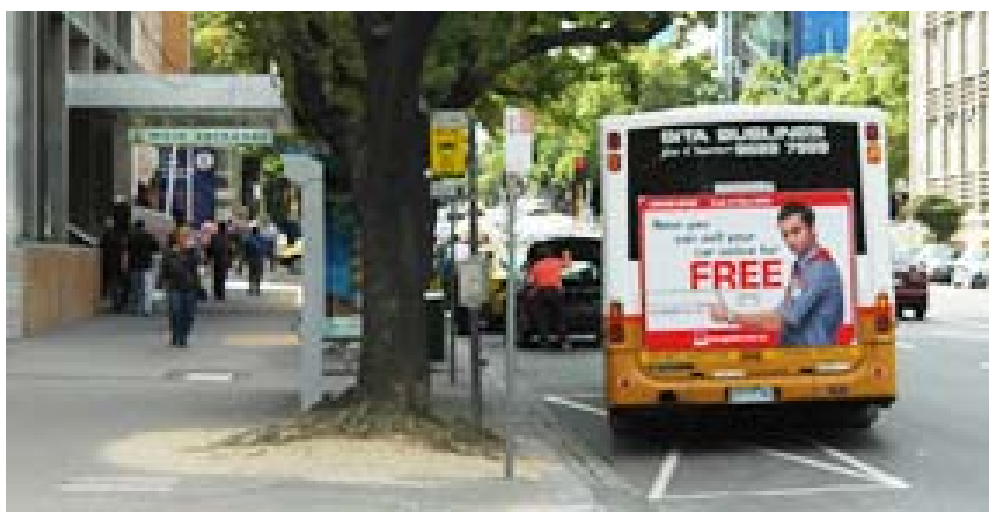
leg of her trip, and during the ride her device collects further offers and books the next leg. Hillary will not come late today. 
The peculiarity of this service is its ad-hoc peer-to-peer communication architecture, realized in a mobile ad-hoc geosensor network. Each node of this network acts as an agent, either as a client agent, having transportation demand, or as a host agent, offering transportation supply. All these nodes are mobile, and all of them are aware of their current location and their route ahead, or at least their destination. Such a service is in sharp contrast to current real-time trip planning services, which are based on central management systems. A current system stores and maintains actual data on the transportation network, i.e., in this case on the location of all vehicles offering transportation, and it performs global route planning for each client in this network. Clearly, central systems are limited in their capacity. They can deal with tracking a few thousand vehicles (see, for example, real-time trip planners of public transportation hosts), but they are not scalable to hundreds of thousands of vehicles of individual traffic in a city. They are also designed for scheduled transportation, and cannot match easily with the autonomy and flexibility of individuals in traffic. Furthermore, in theory every client has to be related to every host, which certainly needs a scalable solution. Current systems do not update a once given response to clients, and they do not consider capacities and current usage of vehicles. The indicated service has the potential to overcome all these limitations.

Trip planning is complex in such networks. The autonomy of hosts makes the transportation network dynamic and unpredictable, which limits the knowledge of any trip planner temporally. Choosing local communication strategies in the communication network limits further the knowledge of a trip planner, this time spatially. On the other side, information about closeby agents is more relevant than that of distant agents. Hence, the trip planner will have to update from time to time its knowledge, and revise its trip plans and bookings. At first, one is interested whether trip planning is possible in this dynamic environment. If so, then the next step is looking for effective trip planning (i.e., a provision of near-to-optimal routes) that is at the same time efficient (i.e., with low communication costs) and elegant (i.e., computationally efficient).

For that purpose we design in this paper a simulation environment. The specification of the design solves the first stage of the trip planning problem: demonstrating that trip planning is possible in an ad-hoc mobile geosensor network. In later work we are looking to approach the second stage of the trip planning problem. Simulation can show effectiveness and efficiency of specific algorithms by statistical measures from larger numbers of simulation runs. 
Without limiting generality we choose the client to be responsible for trip planning, and the transportation hosts to be reactive only. We assume that the client follows a simple wayfinding heuristic, and reduce by that way the problem of trip planning to a pattern matching problem. We will show that in this simplistic case shared ride travelling leads a client always to the destination. That means more powerful algorithms can only improve the defined criteria effectiveness, efficiency, and elegance. However, within the simplistic case we will further compare properties and consequences of different communication strategies, and hence, show a path to approach at least the first two criteria, effectiveness and efficiency.

The paper is structured as follows. In the next section the trip planning aspect of the envisioned service is introduced and discussed in detail. Then we study which information has to be transmitted in the network for successful trip planning. Section 4 compares different communication strategies in geosensor networks for this information exchange. In Section 5 their properties will be investigated by some examples. We will conclude with a summary and future work.

\section{The EnVisioned Service ANd its Position}

\subsection{Current trip planning systems}

Current approaches for real-time individual trip planning and travel assignment are based on central services. This is the case for current commercial solutions, but also for research (Ziliaskopoulos and Mahmassani 1993; Fu 2001; Dillenburg et al. 2002; Chon et al. 2003).

A centralized travel planning system typically consists of a database management system that stores a global view of the transportation network. It keeps track of all changes made by a large number of continuously moving agents: new clients and hosts enter the traffic, hosts get occupied, and agents reach their destinations and disappear from the network. Furthermore, it either plans optimal routes for all clients, or it broadcasts the changes (for example, by Traffic Message Channel, or locally by beacons) to autonomously planning clients (for example, car navigation systems). Since in unpredictable dynamic problems an overall optimum can only be determined in hindsight, the centralized system has to update the travel plans and assignments frequently. With uncertainty in between updates (Dillenburg et al. 2002), some research focuses on the risk in trip planning in dynamic transportation networks (Ran and Boyce 1996; Boyce et al. 1999). If a central system even wants to provide an adjusted optimum for 
all clients, then each change in the network potentially requires updating all trip plans and assignments. Additionally, the system has to manage the communication with clients and hosts. This iterative process is expensive. Since any client is potentially related to any host, it grows exponentially with the number of clients, which means the system is not scalable.

\subsection{Shared ride systems}

Shared ride systems enjoy some popularity in communities such as companies, churches or universities, while public shared ride systems are currently not popular for several reasons. One of them is the association with hitchhiking and its often negative connotation ${ }^{1}$. Another one is the inflexibility of current shared ride systems with real-time travel needs in a dynamic environment.

However, the current situation is surprising given the enormous potential predicted by traffic managers (Dillenburg et al. 2002) or social scientists (Noda et al. 2004; Resnick 2004). Resnick, for example, names some successful shared ride systems that work without any technological support just by social conventions. A more flexible solution, like the proposed one, has therefore the potential for significant social and economic impact.

Hence, shared ride systems have to consider some implications and challenges prior to any realization. They concern, for example, trust and safety, liability, economic incentives and business models, urban mobility and access, fair share (Naor 2005), or privacy. A particular concern is the change of a potentially negative public perception of shared ride travelling, and a change of the proxemics of the involved social beings (Hall 1966). When we look into trip planning we are aware of all these other aspects, but leave them for further work.

\subsection{The envisioned shared ride trip planning system}

Transportation systems are among the proposed applications for mobile sensor networks by Zhao and Guibas (2004). According to a threedimensional characterization of transportation systems (Sussman 2000) our trip planning system is a transportation system which is characterized by individual travellers, urban transport, and private operation (but not private ownership - this is a peer-to-peer service). One of the major functions of transportation systems is travel information, which is provided by telematics and mobile wireless communication (Nijkamp et al. 1996).

\footnotetext{
${ }^{1}$ Note that the connotation of hitchhiking is culturally dependent and not everywhere negative.
} 
The envisioned service goes a step further. It spreads messages on travel demand and transportation supply, plans trips, and books trips in a decentralized peer-to-peer manner. A shared ride trip planning and assignment system consists of basically three related components: (i) shared ride trip planning, (ii) shared ride trip revision, and (iii) shared ride travelling.

The first component itself contains three steps: (i) identifying and formulating a client's transportation need, (ii) searching for optimal shared ride opportunities, and (iii) assigning the providing host to the requesting client. The second component comes into play since the considered network is an unpredictably dynamic one; i.e., plans once made have to be revised from time to time facing previously not known travel opportunities. The component consists of shifted three steps: (i) searching for optimal shared ride opportunities, (ii) assigning the providing host, and (iii) cancelling obsolete previous bookings. The third component is one engaging the physical agents, but can be supported by the services as well. Support during travelling can for example consist of (i) releasing sufficient private information such that the agents can recognize each other at the meeting point, (ii) publishing the travel for security and liability reasons, (iii) rewarding the host with some credits, or (iv) providing a reputation system for hosts and clients based on feedbacks.

The new and challenging aspect of the envisioned service is that is does not need a central service or service provider. It is completely relying on peers (transportation clients and hosts) that are aware of their individual location and travel plans, and are able and willing to communicate. As such they are nodes in a sensor network, where the sensors are geosensors (for positioning), the nodes are mobile, and the communication is radio-based (Stefanidis and Nittel 2004; Zhao and Guibas 2004).

In contrast to most studied problems in geosensor networks, our service requires two-way communication for the negotiation and assignment. Studies in the dissemination of information in geosensor networks (Nittel et al. 2004; Wolfson and Xu 2004) provide basic ideas, but cannot cope with negotiations. Hence we need to search for radio-based communication strategies that efficiently spread messages and efficiently return answers.

\section{DESIGN OF A SHARED RIDE TRIP PLANNING SYSTEM}

In this paper we focus on the first and second component of the service: shared ride trip planning and trip revision. In principle we apply the following service design: Hillary's device formulates and broadcasts a request into a peer-to-peer communication network, collects all offers from hosts 
responding to this request, selects the optimal offer for the intended trip, and books it. Eventually previous bookings have to be cancelled. In this design the hosts are reactive: they respond to requests from clients according to their own plans and capacities, they forward these requests and they accept bookings from clients. For that purpose they maintain a list of their travel assignments.

Note that other designs are possible as well. In particular the hosts could have a more proactive role, for example, providing a distributed database of current transportation supply (Harwood and Tanin 2003), or actively harvesting for clients. We leave the question for an optimal design (in terms of a most efficient and effective trip planning) for future work. Currently we are satisfied if a service of the proposed design can solve the trip planning problem.

Now, within our chosen design we have to specify some aspects in more detail: (i) how the clients can collect the information of current transportation supply, (ii) how they can do trip planning on this information, and (iii) how they can book selected offers. In this sequence (i) and (iii) form the communication aspect, and (ii) forms the client's internal computation aspect.

\section{SHARED RIDE TRIP PLANNING}

To preserve generality let us assume that hosts travel autonomously. They do not announce their travel prior to their start, they have their individual travel plan (a route), and this travel plan can have any form, including stops, being non-shortest, containing cycles, or travelling forth and back. Let us further assume that hosts, although willing to take passengers, are not willing to make detours for these passengers. This is a restriction that limits generality (for example, a tram could not make a detour, but a taxi could). But since reality is more flexible than this assumption, we still can deliver a valid proof that trip planning is possible.

Furthermore, in practice the travel plans of hosts adapt to traffic conditions. That means that hosts can publish their current and future positions - which basically is a trajectory of a mobile object (Güting et al. 2000) - but the future positions are temporally uncertain (Ran and Boyce 1996; Boyce et al. 1999; Dillenburg et al. 2002).

In summary, at a specific point in time we can maximally know which hosts are currently travelling, where they are, what their current booking status is, and what their travel intentions are. We can not know with cer- 
tainty the future positions of currently traveling hosts, their future booking states, and we cannot see which new hosts will enter traffic next. With other words, at any point in time we can determine an optimal route, but in hindsight this might not have been the optimal one.

Let us consider a client's travel demand. A client is interested to reach a destination, and the trip shall be optimal in terms of a cost function. The cost function depends on the client's context, and may consider, for example, travel time, trip fare, number of transfers, or reputation of hosts. Without limiting generality we choose in our simulation travel time.

In this scenario, a client's request can be relatively unspecific, consisting only of current position and destination. Then each host has a relatively complex task (requiring knowledge of the full street network) to determine whether its own route could contribute to a client's request such that the host could make an offer. Hence, for the time being let us assume that the client's request will be specific, consisting of the current position, the destination, and a route or a set of alternative routes. This could be a preferred route, or the $k$ routes of shortest length (Yen 1971). Note that neither of these routes may lead the fastest trip. Hence this is a heuristic. Then each host has only to compare the requested street segments with its own route, and will make an offer for all segments in the intersection set. Note that these segments still can be unreachable for the client since so far the temporal aspect was not considered. It is the client, which collects all offers and constructs a dynamic network. Each offer forms one or several timedependent links in this network.

In principle, a time-dependent shortest path algorithm (Cooke and Halsey 1966) can find an optimal route in this network and identify the contributing host offers. However, the constructed dynamic network is not necessarily connected, and it might also not contain the destination. Hence, the algorithm can only compute a shortest path tree from the start node, select one branch, and identify the contributing host offers of this branch.

Since clients and hosts are moving in a dynamic environment, plans and booking have to be revised from time to time. In these iterations, a client will also fill the gaps to its destination. In summary, we have shown that route planning in a dynamic environment is in principle possible, computationally efficient, and can be as effective as temporally limited knowledge allows. A detailed example will be given in Section 6. 
Communication consists of three steps: a request from a client, offers from hosts, and booking or cancellation messages from the client. Since the communication technology is short-range radio-based (Zhao and Guibas 2004), all types of messages are broadcasted, and are received only by agents within radio range. If messages shall be sent further they have to be forwarded by multi-hop communication, i.e. repeated broadcasting by other nodes in larger distance. In any case messages can only reach agents that are in a direct (single-hop) communication distance, or in multi-hop communication range.

Geosensor networks try to optimize energy consumption of agents. By far the largest energy is needed for broadcasting (compared to listening, computing, or sleeping). Hence, geosensor networks communicate in synchronized (short) communication time frames, and go into a sleep or processing mode in the meantime. Additionally, considering a very large number of agents in a geosensor network, network bandwidth becomes a problem. Since all agents potentially could communicate continuously at the same time, collisions on the channels, limited capacity of the channels, and packet loss are more common, and thus, communication is less reliable. Thus, since broadcasting is expensive, it makes sense to study the number of messages to be sent in the previous scenario, and to optimize it. Another reason to do so is the explosion of messages in the network if every client floods the whole network with a request.

The following thought might help to actively limit communication to a local neighbourhood only. The hosts in the neighbourhood of a client can soon be at the client's position. Their potential offer is more important for an optimal trip of the client than potential offers from far hosts. Furthermore, the client is going to update a once made travel plan regularly. Hence, a host that is far at one point in time, but relevant for an optimal solution, will become closer and is at some time within a short range of the client. That means, by limiting a communication to a local neighborhood the worst case that can happen is that the client starts travelling with a suboptimal host, which might increase the number of transfers but not the total travel time.

Different communication strategies in geosensor networks are investigated elsewhere (Nittel et al. 2004; Wolfson and Xu 2004). However, these investigations concentrate on the spreading of information through a network of agents, but not on negotiations. Instead, in our scenario we have to realize two-way communication, which behaves fundamentally different. First, 
all the communication caused by a single request has to occur in one communication window (we will call it a negotiation cycle). This is a consequence of the activity/sleep mode of the individual agents. Practically this requirement limits as well realizations to local neighbourhoods. Secondly, secondary messages (offers, bookings and cancellations) should be designed such that they can be directed to further reduce their spreading.

In the following section we explain in a simple example the negotiation cycle for a single host.

\section{A NEGOTIATION EXAMPLE}

Consider the simplified scenario of Figure 1. In a street grid network, a client $\boldsymbol{c}$ formulates at time $t_{0}$ a request for a trip A2-B2-C2. The request is broadcasted and received by all agents in radio range, which means within the direct neighborhood of the client. Host $\boldsymbol{h}$ is in range, receives the request, and matches the requested trip with its own travel plans (A1 $\left(t_{0}\right)$ A2 $\left(t_{1}\right)$-B2 $\left.\left(t_{2}\right)-\mathrm{B} 3\left(t_{3}\right)\right)$. Note that hosts have time-stamped travel plans. For the intersection of the two trips, $\boldsymbol{h}$ makes an offer of the form [h, A2-B2, $t_{1}$ ]. Client $\boldsymbol{c}$ collects all offers (here only one), selects one (starting earliest), and books the host by a message $\left[\boldsymbol{c}, \boldsymbol{h}, \mathrm{A} 2-\mathrm{B} 2, \boldsymbol{t}_{1}\right]$. Host $\boldsymbol{h}$ will reserve a seat for $\boldsymbol{c}$ for that segment. The negotiation cycle ends here, a travel cycle follows. After some time, e.g., after traveling one street segment, $\boldsymbol{c}$ will initiate a new negotiation cycle.

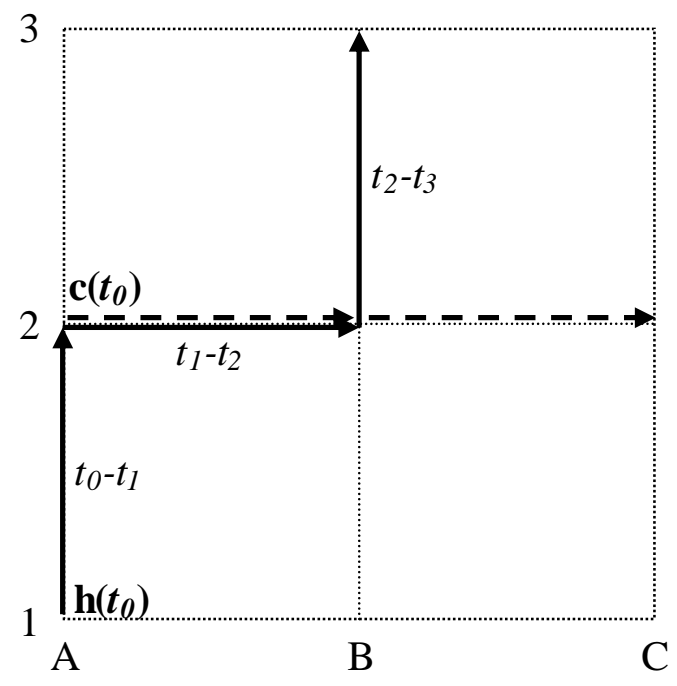

Figure 1: A client $c$ and a host $h$ at $t_{0}$.

This simple schema is sufficient for a simulation with large numbers of hosts and clients. In a simulation both of the discussed aspects can be in- 
vestigated: the heuristics applied by hosts to predetermine a route, and the depth to which messages are spread by multi-hopping.

\section{Conclusions}

We have investigated and demonstrated that shared ride trip planning in geosensor networks is possible, and we have proposed (but not yet investigated) how route heuristics and short range communication strategies can effectively and efficiently solve the trip planning problem. We further have proposed a simulation environment to investigate and assess these ideas in detail.

In the meantime we have implemented a simulation environment, and test various alternatives for identifying optimal strategies. Results will soon be published. However, the sketched simulation environment can also be extended in various directions, to consider additionally factors such as nongridded street networks, or multiple clients and their competition for transportation supply. In principle the simulation environment could also be used for testing the consequences of individual behaviour and preferences, such as mutual interest in client and host reputation.

\section{ACKNOWLEDGEMENTS}

The initial idea of a peer-to-peer shared ride service was born in a conversation with Monika Sester, Hannover, which took place in a coffee house in Palma, Mallorca, after AGILE 2002.

\section{REFERENCES}

Boyce, D.E.; Ran, B.; Li, I.Y., 1999: Considering Travelers' Risk-Taking Behavior In Dynamic Traffic Assignment. In: Bell, M.G.H. (Ed.), Transportation Networks: Recent Methodological Advances, Elsevier Science, Oxford, pp. 67-81.

Chon, H.D.; Agrawal, D.; Abbadi, A.E., 2003: FATES: Finding A Time Dependent Shortest Path. In: Chen, M.S. (Ed.), MDM 2003. Lecture Notes in Computer Science, 2574. Springer, Berlin, pp. 165-180.

Cooke, K.L.; Halsey, E., 1966: The Shortest Route through a Network with Time-Dependent Internodal Transit Times. Journal of Mathematical Analysis and Applications, 14: 493-498. 
Dillenburg, J.F.; Wolfson, O.; Nelson, P.C., 2002: The Intelligent Travel Assistant, 5th International Conference on Intelligent Transportation Systems. IEEE, Singapore, pp. 691-696.

Fu, L., 2001: Adaptive Routing Algorithm for In-Vehicle Route Guidance Systems with Real-Time Information. Transportation Research Part B, 35 (8): 749-765.

Güting, R.H.; Böhlen, M.H.; Erwig, M.; Jensen, C.S.; Lorentzos, N.A.; Schneider, M.; Vazirgiannis, M., 2000: A foundation for representing and querying moving objects. ACM Transactions on Database Systems, 25 (1): 1-42.

Hall, E.T., 1966: The Hidden Dimension. Doubleday \& Company, Garden City, NY.

Harwood, A.; Tanin, E., 2003: Hashing Spatial Content over Peer-to-Peer Networks. In: Zukerman, M. (Ed.), Proceedings of the Australian Telecommunications, Networks, and Applications Conference, Melbourne.

Naor, M., 2005: On Fairness in the Carpool Problem. Journal of Algorithms, 55: 93-98.

Nijkamp, P.; Pepping, G.; Banister, D., 1996: Telematics and Transport Behaviour. Advances in Spatial Science. Springer, Berlin.

Nittel, S.; Duckham, M.; Kulik, L., 2004: Information Dissemination in Mobile Ad-hoc Geosensor Networks. In: Egenhofer, M.; Freksa, C.; Miller, H.J. (Eds.), Geographic Information Science. Lecture Notes in Computer Science, 3234. Springer, Berlin, pp. 206-222.

Noda, I.; Ohta, M.; Shinoda, K.; Kumada, Y.; Nakashima, H., 2004:

Evaluation of Usability of Dial-a-Ride Systems by Social Simulation. In: Hales, D. et al. (Eds.), Multi-Agent-Based Simulation III. Lecture Notes in Artificial Intelligence, 2927. Springer, Berlin, pp. 167-181.

Ran, B.; Boyce, D., 1996: Modeling Dynamic Transportation Networks. Springer, Berlin.

Resnick, P., 2004: Impersonal Sociotechnical Capital, ICTs, and Collective Action Among Strangers. In: Dutton, W. et al. (Eds.), Transforming Enterprise, MIT Press, Cambridge, Mass., pp. 399-412.

Stefanidis, A.; Nittel, S., 2004: GeoSensor Networks. CRC Press, Boca Raton, FL.

Sussman, J., 2000: Introduction to Transportation Systems. Artech House, Boston, MA. 
Wolfson, O.; Xu, B., 2004: Opportunistic Dissemination of SpatioTemporal Resource Information in Mobile Peer-to-Peer Networks, First International Workshop on P2P Data Management, Security and Trust. DEXA, Zaragoza, Spain, pp. 954-958.

Yen, J.Y., 1971: Finding the $k$ shortest loopless paths in a network. Management Science, 17 (11): 712-716.

Zhao, F.; Guibas, L.J., 2004: Wireless Sensor Networks. Elsevier, Amsterdam.

Ziliaskopoulos, A.; Mahmassani, H., 1993: A Time-Dependent Shortest Path Algorithm for Real-Time Intelligent Vehicle Highway System Applications. Transportation Research Record (1408): 94-104. 


\section{University Library}

\section{- M M I E R R A A gateway to Melbourne's research publications}

Minerva Access is the Institutional Repository of The University of Melbourne

Author/s:

Nittel, S;Winter, S;Nural, A;Cao, T

Title:

Shared ride trip planning with geosensor networks

Date:

2007-01-01

Citation:

Nittel, S., Winter, S., Nural, A. \& Cao, T. (2007). Shared ride trip planning with geosensor networks. Miller, HJ (Ed.). GeoJournal Library, (1), 88, pp.179-194. SPRINGER.

Publication Status:

Published

Persistent Link:

http://hdl.handle.net/11343/33839 